\title{
Weak Fault Detection for Rolling Bearings in Varying Working Conditions through the Second-Order Stochastic Resonance Method with Barrier Height Optimization
}

\author{
Huaitao Shi, ${ }^{1}$ Yangyang Li $\left(\mathbb{D},{ }^{1}\right.$ Peng Zhou $\mathbb{D}^{1},{ }^{1}$ Shenghao Tong $\mathbb{D}^{1},{ }^{1}$ Liang Guo, ${ }^{2}$ \\ and Baicheng $\mathrm{Li}^{3}$ \\ ${ }^{1}$ School of Mechanical Engineering, Shenyang Jianzhu University, Shenyang, Liaoning 110168, China \\ ${ }^{2}$ Southwest Jiaotong University, Chengdu, Sichuan 611700, China \\ ${ }^{3}$ University of Washington, Seattle, WA 98195, USA
}

Correspondence should be addressed to Peng Zhou; zhoupeng@sjzu.edu.cn and Shenghao Tong; tongshenghao@sjzu.edu.cn

Received 3 March 2021; Revised 21 May 2021; Accepted 4 June 2021; Published 16 June 2021

Academic Editor: Paola Forte

Copyright (c) 2021 Huaitao Shi et al. This is an open access article distributed under the Creative Commons Attribution License, which permits unrestricted use, distribution, and reproduction in any medium, provided the original work is properly cited.

\begin{abstract}
The stochastic resonance (SR) method is widely applied to fault feature extraction of rotary machines, which is capable of improving the weak fault detection performance by energy transformation through the potential well function. The potential well functions are mostly set fixed to reduce computational complexity, and the SR methods with fixed potential well parameters have better performances in stable working conditions. When the fault frequency changes in variable working conditions, the signal processing effect becomes different with fixed parameters, leading to errors in fault detection. In this paper, an underdamped second-order adaptive general variable-scale stochastic resonance (USAGVSR) method with potential well parameters' optimization is put forward. For input signals with different fault frequencies, the potential well parameters related to the barrier height are figured out and optimized through the ant colony algorithm. On this basis, further optimization is carried out on undamped factor and step size for better fault detection performance. Cases with diverse fault types and in different working conditions are studied, and the performance of the proposed method is validated through experiments. The results testify that this method has better performances of weak fault feature extraction and can accurately identify different fault types in the input signals. The method proves to be effective in the weak fault extraction and classification and has a good application prospect in rolling bearings' fault feature recognition.
\end{abstract}

\section{Introduction}

Rotary machines are generally applied to modern industrial production, and rolling bearings play a key role in rotary machines [1-3]. Unexpected rolling bearing failures lead to machine failures and shutdowns, causing huge economic losses and even threatening the personal safety of workers [4-6]. Therefore, weak fault feature recognition and fault detection of bearings are very important [7-9].

The information on the running states of rolling bearings is contained in vibration signals. In recent years, many scholars have used the vibration signals of rolling bearings for fault diagnosis [10-13]. However, information on fault characteristics is often obscured by equipment operation noise and environmental background noise, and it is difficult to extract weak faults of bearings $[14,15]$. Therefore, some scholars put forward some methods to analyze weak characteristic signals, such as empirical mode decomposition, wavelet transform, and time-frequency analysis [16-18]. These methods can extract the characteristics of weak signals by eliminating or suppressing noise. In this process, the weak fault information of rolling bearings is possible to be suppressed. According to the limitations of the above methods, Zhang and Zhou [19] proposed the ensemble empirical mode decomposition method, which introduced noise to deal with the mode aliasing problem, so as to realize the fault feature extraction. Noise acts as a kind of energy signal, and the stochastic resonance method improves the detection 
performance of weak faults by converting noise energy into weak signals and then realizes fault feature extraction of bearings $[20,21]$.

In recent years, the SR method has been widely applied in fault feature extraction and recognition of bearings $[22,23]$. Li and Shi [24] introduced a new piecewise nonlinear SR to enhance early fault features of machinery. He et al. [25] analyzed the multiscale SR spectral method, which studied the time-frequency distribution of the signal and used its scale as a modulation system to recognize the fault characteristics of rolling bearings. Li et al. [26] introduced a new adaptive SR method, which improved the detection performance of weak faults by using indicators such as correlation coefficient. Lei et al. [27] made use of the steady state for matching, and an underdamped SR was proposed, which had a good enhancement capability for weak signals by suppressing multiscale noise. Li et al. [28] introduced a delay feedback term into the overdamped monostable system, which can availably use history information of the input signal to intensify the influence on the resonance phenomenon. By selecting appropriate underdamping factor and calculating step size, $\mathrm{Lu}$ et al. [29] introduced an underdamped variable step-size second-order SR method, and output signal-to-noise ratio (SNR) is increased.

In order to simplify the complexity of the system and increase computational efficiency, most of the existing SR methods fix the parameters as specific values. The potential barrier is crucial in the output of the SR system. The optimal potential barrier corresponds to the optimal output of the system. However, different input signals have different dominant barriers, and fixed SR barrier parameters limit the system to achieving the optimal output. Optimizing the barrier height is the basis for the system to achieve the optimal output. Therefore, an underdamped second-order adaptive general variable-scale stochastic resonance method with the optimization of potential well parameters is introduced for bearing fault detection. According to different input signals, the optimal parameters of the system are adaptively matched by optimization algorithm, and optimal barriers corresponding to different input signals are obtained, respectively. On the basis of the most dominant barrier, the optimal matching of noise, the input signal, and the nonlinear system are realized, and the weak fault features of bearings are recognized.

The following research contents are as follows: in the second part, the USAGVSR diagnosis method is introduced. The third part carries on the simulation. In the fourth part, cases of diverse fault types in rolling bearings are studied. In the fifth part, the rolling bearing faults under different working conditions are studied, and the accuracy of the proposed method is verified. The sixth part is the result and discussion of this paper. The seventh part draws the conclusion of this paper.

\section{Method}

2.1. Underdamped Second-Order Adaptive General VariableScale Stochastic Resonance. The classical model of SR is a bistable system. The dynamic equation of bistable SR is [29]

$$
\frac{\mathrm{d} x(t)}{\mathrm{d} t}=-\frac{\mathrm{d} U(x)}{\mathrm{d} x}+S(t)+N(t)
$$

where $S(t)$ represents the weak periodic signal to be detected, $N(t)$ represents the Gaussian white noise, $U(x)$ is the potential function, and $x(t)$ represents the oscillation trajectory of the particle. $U(x)$ is expressed as

$$
U(x)=-\frac{a}{2} x^{2}+\frac{b}{4} x^{4}
$$

where $a$ and $b$ are potential well parameters, respectively; the barrier height is $\Delta U=\left(a^{2} / 4 b\right) . N(t)$ is expressed as

$$
\left\{\begin{array}{l}
\langle N(t)\rangle=0 \\
\langle N(t) N(0)\rangle=2 D \delta(t)
\end{array}\right.
$$

where $D$ is the noise intensity, $\delta(t)$ is the Dirac function, and $<>$ is the statistical average operator. The weak signal $S(t)$ to be detected is denoted as

$$
S(t)=A \cos (2 \pi f t+\varphi) .
$$

The output effect of SR depends on the transition of the Brownian particle in the potential well. The motion diagram of the Brownian particle in the SR system is shown in Figure 1. As shown in Figure 1(a), the Brownian particle is initially located in the left potential well. Under the excitation of the weak periodic signal and the noise, the particle transitions from the left potential well to the right potential well, as shown in Figure 1(b). The Brownian particle is in a stable state, located in the right potential well, as shown in Figure 1(c). Due to the joint excitation of the weak periodic signal and the noise, the Brownian particle again generates a transition, jumping from the right potential well to the left potential well, as shown in Figure 1(d).

As shown in Figure 1, the barrier height affects the transition frequency of the Brownian particle. When the barrier height is too high, the Brownian particle cannot obtain enough energy to generate the transition in the left and right potential wells. When the barrier height is too low, the Brownian particle jumps violently in the left and right potential wells and moves irregularly. In this case, the SR energy distribution is not enough to meet the demand of weak fault feature extraction. When the transition frequency of the Brownian particle is consistent with the input signal frequency, the output effect of SR is the best. Under different working conditions, the frequency of the input signal changes. In order to achieve the best output effect of SR, the transition frequency of the Brownian particle is changed by optimizing the barrier height so that the transition frequency of the Brownian particle is consistent with the input signal frequency. Optimizing the barrier height is the basis for the system to achieve the optimal output.

In practical engineering applications, underdamping phenomenon is common, and the damping factor of the SR system will affect the output response of the system. Therefore, considering the damping factor of the system, the model of SR is expressed as

$$
\frac{\mathrm{d}^{2} x(t)}{\mathrm{d} t^{2}}=-\frac{\mathrm{d} U(x)}{\mathrm{d} x}-k \frac{\mathrm{d} x(t)}{\mathrm{d} t}+S(t)+N(t),
$$

where $k$ represents the underdamping factor. Substitute equations (2)-(4) into equation (5) and convert into 


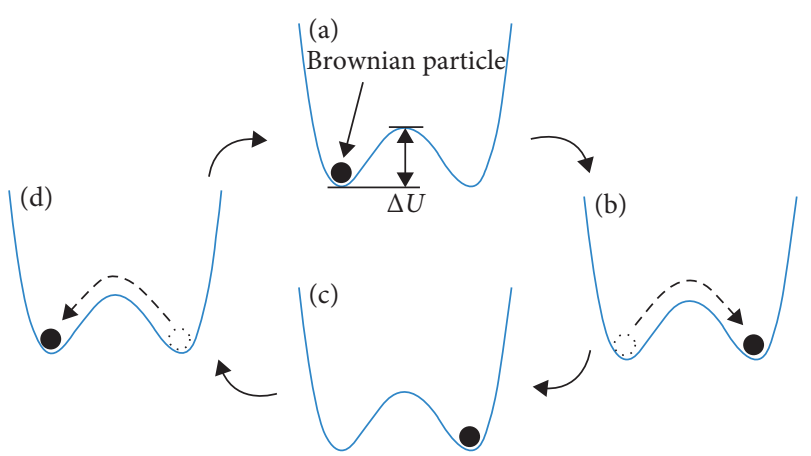

Figure 1: The motion diagram of the Brownian particle.

$\frac{\mathrm{d}^{2} x(t)}{\mathrm{d} t^{2}}=a x(t)-b x(t)^{3}-k \frac{\mathrm{d} x(t)}{\mathrm{d} t}+A \cos (2 \pi f t+\varphi)+\sqrt{2 D} \xi(t)$.

Classical SR is suitable for small parameters, but it is a high-frequency signal in engineering practice, so the general variable-scale stochastic resonance model is used to make it suitable for the high-frequency weak signal, and substitution variables are introduced [30]:

$$
\begin{aligned}
x(t) & =z(\tau), \\
\tau & =m t,
\end{aligned}
$$

where $m$ is the scale coefficient. Substitute equation (7) into equation (6) to get

$$
\begin{aligned}
\frac{\mathrm{d}^{2} z(\tau)}{\mathrm{d} \tau^{2}}= & \frac{a}{m^{2}} z(\tau)-\frac{b}{m^{2}} z^{3}(\tau)-\frac{k}{m} \frac{\mathrm{d} z(\tau)}{\mathrm{d} \tau} \\
& +\frac{A}{m^{2}} \cos \left(2 \pi \frac{f}{m} \tau+\varphi\right)+\sqrt{\frac{2 D}{m^{3}}} \xi(\tau) .
\end{aligned}
$$

Make $\left(a / m^{2}\right)=a_{1}, \quad\left(b / m^{2}\right)=b_{1}, \quad(k / m)=k_{1}$, $(f / m)=f_{1}, \quad\left(A / m^{2}\right)=A_{1}, \quad$ and $\quad\left(\sqrt{D} / m^{2}\right)=\sqrt{D_{1}}, \quad$ and substitute them into equation (8):

$$
\begin{aligned}
\frac{\mathrm{d}^{2} z(\tau)}{\mathrm{d} \tau^{2}}= & a_{1} z(\tau)-b_{1} z^{3}(\tau)-k_{1} \frac{\mathrm{d} z(\tau)}{\mathrm{d} \tau} \\
& +A_{1} \cos \left(2 \pi f_{1} \tau+\varphi\right)+\sqrt{2 D_{1} m} \xi(\tau) .
\end{aligned}
$$

Equation (9) is the general variable-scale form of the underdamped bistable system in equation (6), equation (9) shows that, after dealing with the variable scale, the signal frequency $f_{1}$ is changed to $1 / m$ of the original frequency, the conversion between high and low frequency is realized by selecting an appropriate $m$ value, and $S(t)$ and $N(t)$ are changed to $1 / \mathrm{m}^{2}$ of the original signal, it can meet the requirements of classical SR for small parameters.

The output of the USAGVSR system is complex and difficult to be analyzed by the analytical method. Therefore, the fourth-order Runge-Kutta is applied to numerical analysis. The expression of iterative algorithms is as follows:

$$
\left\{\begin{array}{l}
y_{1}=y[i] \\
x_{1}=-U^{\prime}(x[i])-k y_{1}+S[i]+N[i], \\
y_{2}=y[i]+x_{1} \frac{h}{2} \\
x_{2}=-U^{\prime}\left(x[i]+y_{1} \frac{h}{2}\right)-k y_{2}+S[i]+N[i] \\
y_{3}=y[i]+x_{2} \frac{h}{2}, \\
x_{3}=-U^{\prime}\left(x[i]+y_{2} \frac{h}{2}\right)-k y_{3}+S[i+1]+N[i+1] \\
y_{4}=y[i]+x_{3} h, \\
x_{4}=-U^{\prime}\left(x[i]+y_{3} h\right)-k y_{4}+S[i+1]+N[i+1]
\end{array}\right.
$$

where $y=(\mathrm{d} x / \mathrm{d} t), x[i], S[i]$, and $N[i]$ are discrete representations of $x(t), S(t)$, and $N(t)$, respectively, and $i=1,2, n, n$ represents lengths of discrete sampled data, and $h$ represents the calculation step size.

For equation (6), let $y(t)=(\mathrm{d} x(t) / \mathrm{d} t)$, and get

$$
\frac{\mathrm{d} y(t)}{\mathrm{d} t}=a x(t)-b x(t)^{3}-k y(t)+A \cos (2 \pi f t+\varphi)+\sqrt{2 D} \xi(t) .
$$

Make $(\mathrm{d} x(t) / \mathrm{d} t)=0,(\mathrm{~d} y(t) / \mathrm{d} t)=0, A=0$, and $D=0$, and the three singularities of the system are obtained: $\left(x_{+}, y_{+}\right)=(\sqrt{(a / b)}, 0), \quad\left(x_{0}, y_{0}\right)=(0,0)$, and $\left(x_{-}, y_{-}\right)=$ $(-\sqrt{a / b}, 0)$.

The output SNR is [31]

$$
\operatorname{SNR}=\frac{\int_{0}^{\infty} P_{S}(i) \mathrm{d} i}{P_{N}(i=2 \pi f)},
$$

where $P_{S}(i)$ represents power spectra of the signal and $P_{N}(i)$ represents power spectra of noise.

\subsection{Fault Identification with Optimization of Potential Well} Parameters. In SR systems, the barrier height determines the output of SR. For different input signals, the corresponding most advantageous barrier is different. Therefore, it is very important to determine the most advantageous barriers for different input signals in practical engineering 
applications. In this paper, the bistable adaptive general variable-scale stochastic resonance model and ant colony optimization algorithm are applied to find the most advantageous well parameters $a_{1}$ and $b_{1}$ corresponding to different input signals, and the optimal potential barriers are found. The SNR is applied to evaluate the output of the system, and SNR is expressed as

$$
\left\{\begin{array}{l}
\operatorname{SNR}=10 \lg \frac{P_{1}(f)}{P_{2}(f)}, \\
P_{1}(f)=2|X(f)|^{2}, \\
P_{2}(f)=\frac{2}{n}\left(\sum_{k=1}^{n / 2}\left|X\left(f_{k}\right)\right|^{2}-P_{1}(f)\right),
\end{array}\right.
$$

where $X\left(f_{k}\right)$ represents the discrete Fourier transform of the corresponding signal sequence $X(n), P_{1}(f)$ represents the signal energy, and $P_{2}(f)$ represents the noise energy.

The steps of ant colony algorithm are as follows [32]:

(1) Initialize system parameters, including the number of ants $m_{1}$, pheromone concentration $T$, maximum iteration number $N C \_$max, and termination condition $e$.

(2) Place $m_{1}$ ants randomly on the initial node, constantly modify the information of nodes with ants, calculate SNR, and update pheromone based on the SNR.

(3) Find the maximum probability and judge whether $e$ is satisfied. If it is, end the program; if not, return to Step (2).

(4) Output the maximum SNR and optimal parameters $a_{1}$ and $b_{1}$ of the system.

For different input signals, the ant colony algorithm is applied to match the corresponding most advantageous barrier adaptively by calculating SNR, and the optimal parameters $a_{1}$ and $b_{1}$ are determined. On this basis, the signals are processed by the underdamped second-order stochastic resonance (USSR) system, and then ant colony optimization algorithm is applied to search for optimal underdamped factor and step size, so as to achieve the optimal system response. The USAGVSR method with potential well parameters' optimization is shown in Figure 2.

The potential barrier plays an important role in the output of the SR system, and the optimal potential barrier corresponds to the optimal output of the system. For different input signals, the corresponding most advantageous barrier is different. Optimization of potential well parameters is the basis of SR system processing. The steps of the USAGVSR method with potential well parameters' optimization are as follows: vibration signals of rolling bearings are collected by vibration sensors, and the collected vibration signals are preprocessed. The underdamping factor in equation (9) is equal to 0 , the adaptive general variable-scale stochastic resonance system model is obtained and used to process vibration signals, and the parameters are optimized by ant colony algorithm. The SNR is used to quantify the optimization results to confirm whether the cutoff conditions of optimization iteration are met. If not, the local search range of parameters $a$ and $b$ is changed by using the tabu table, and then SNR is calculated again to optimize the parameters. If the cutoff condition is satisfied, that is, when a certain number of iterations are reached, SNR tends to be stable, the optimal barriers corresponding to the vibration signals are obtained, and the optimal parameters $a_{1}$ and $b_{1}$ are output. Then, the vibration signals are input to the underdamped second-order stochastic resonance system and take advantage of the corresponding most advantageous barriers, and the system parameters are initialized. The optimum SNR is searched by the optimization algorithm, and then the optimal output parameters are obtained. The corresponding frequency domain graphs of vibration signals are obtained, the weak fault features are extracted, and the fault types are identified.

\section{Simulation}

To confirm the effectiveness of the proposed method, three analog signals are introduced to analysis parameters. The analog signal is $X_{i}=A_{i} \cos \left(2 \pi f_{i} t\right)$, where $i=1,2$, and 3 . And the amplitude $A_{1}=0.1 ; A_{2}=0.5 ; A_{3}=1$, frequency $f_{1}=50 \mathrm{~Hz} ; f_{2}=100 \mathrm{~Hz}$; and $f_{3}=150 \mathrm{~Hz}$, and Gaussian white noise is added to the analog signals, respectively. For given different potential well parameters $a_{1}$ and $b_{1}$, Figure 3 shows the corresponding potential barriers of the system.

Figure 3 shows that when the parameters $a_{1}$ and $b_{1}$ are different, the height and width of the barrier corresponding to the system are also different, and the Brownian particle also has different transition frequencies. Therefore, for different input signals, it is important to determine the most dominant barriers and the best potential well parameters in order to achieve the optimal system output.

For three different analog signals, the optimal SNR is searched by the ant colony algorithm, respectively, the most dominant barriers corresponding to the analog signals are obtained, and the best potential well parameters $a_{1}$ and $b_{1}$ are obtained. For analog signal $X_{1}$, the optimal barrier height $\Delta U$ is 0.017 , and the corresponding optimal potential well parameters $a_{1}=0.241$ and $b_{1}=0.852$. Set fixed potential well parameters $a_{2}=1$ and $b_{2}=1$. For the optimal and fixed potential well parameters, the SNR change of analog signal $X_{1}$ with the increase of $D$ is shown in Figure 4 .

maxl represents the maximum SNR corresponding to the analog signal in the case of the optimal potential well parameters, and max2 represents the maximum SNR corresponding to the analog signal in the case of the fixed potential well parameters. Similarly, for analog signal $X_{2}$, the optimal barrier height $\Delta U$ is 0.002 , and the optimal potential well parameters $a_{1}=0.076$ and $b_{1}=0.754$. For analog signal $X_{3}$, the optimal barrier height $\Delta U$ is 0.060 , and the optimal potential well parameters $a_{1}=0.364$ and $b_{1}=0.958$. Figure 5 shows the SNR changes of analog signals $X_{2}$ and $X_{3}$ with the increase of $D$.

The figures show that the most dominant barriers corresponding to different analog signals are different, and the most dominant well parameters are also different. The 


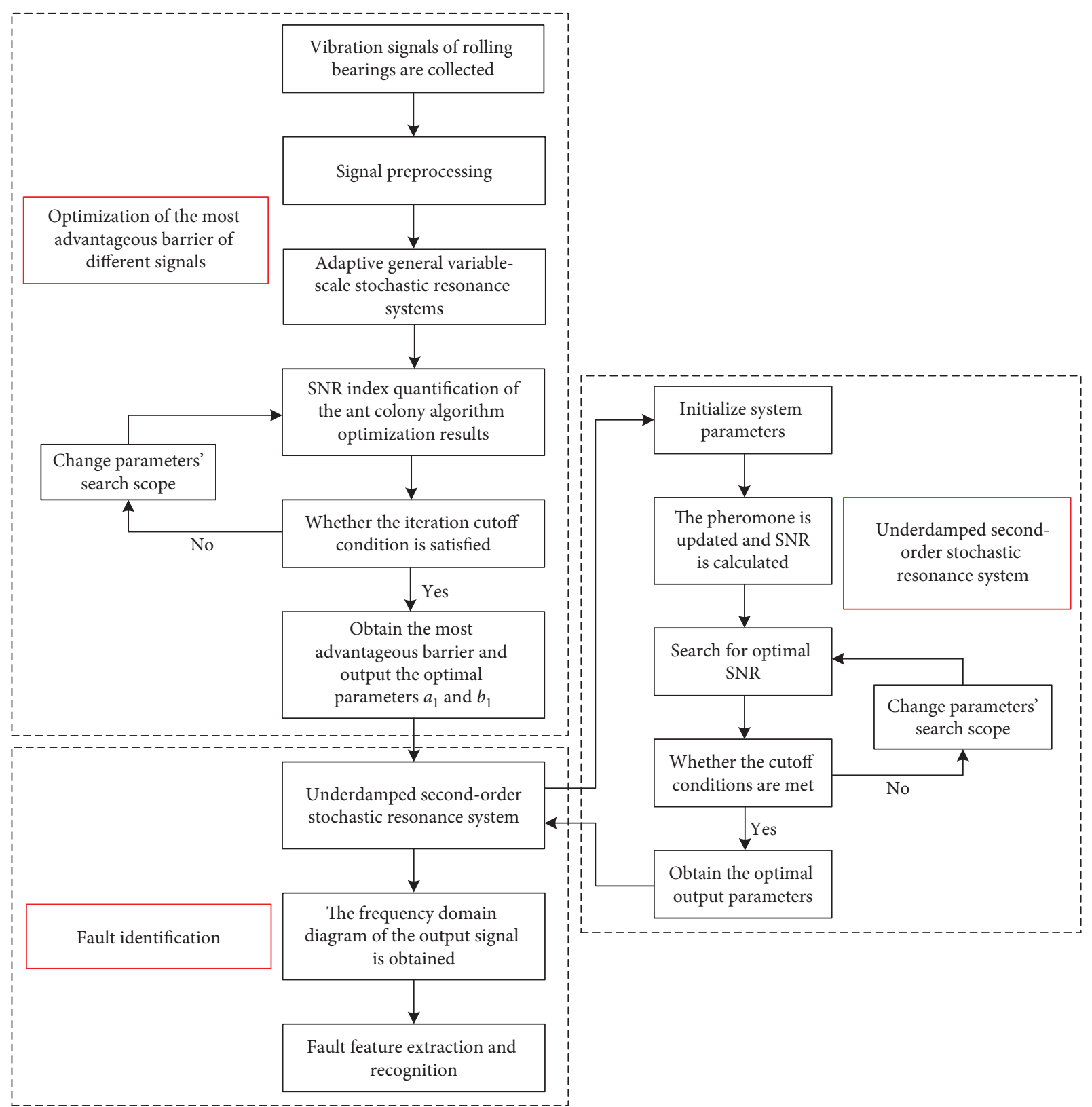

FIGURE 2: The USAGVSR method with potential well parameters' optimization.

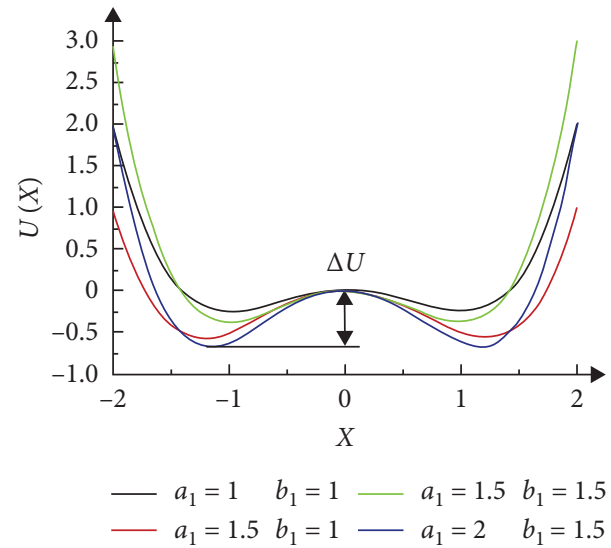

Figure 3: Barriers for different well parameters. 


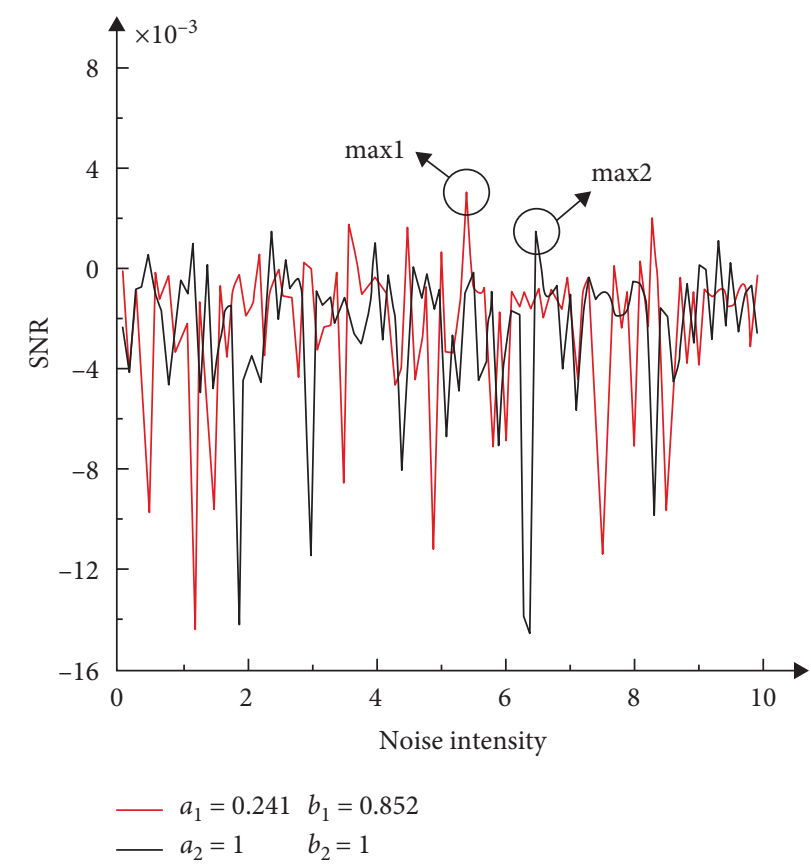

FIGURE 4: SNR variation of analog signal $X_{1}$.

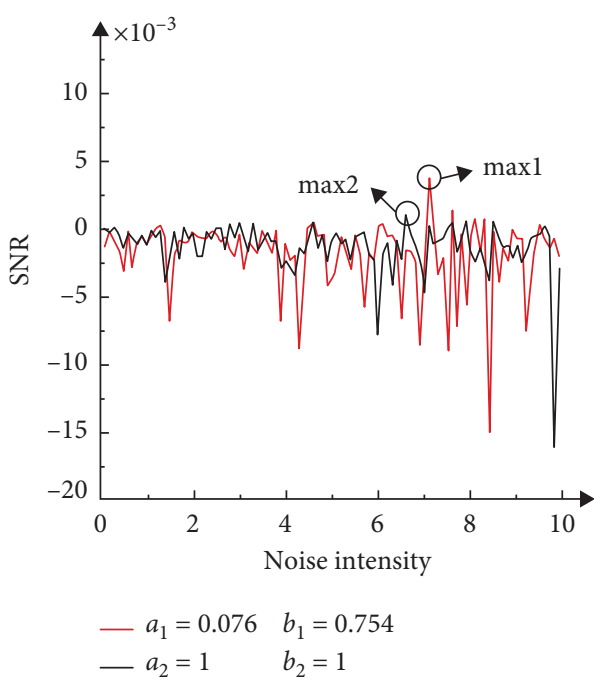

(a)

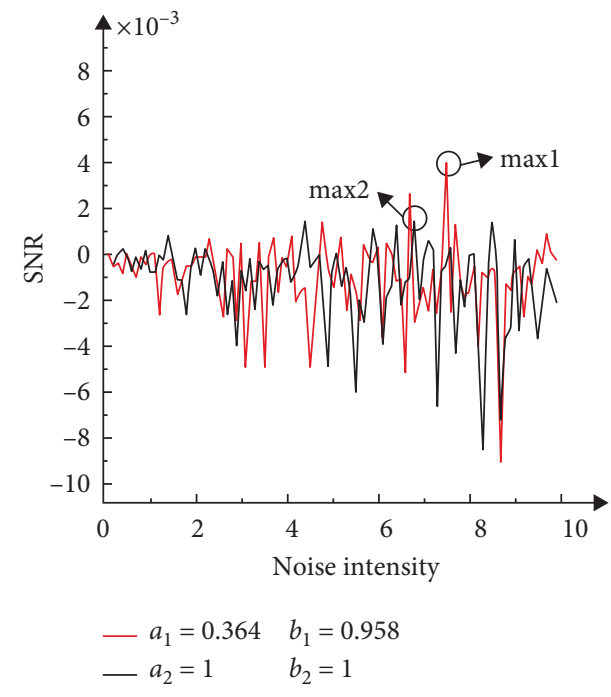

(b)

FIgURE 5: SNR variation of (a) analog signal $X_{2}$ and (b) analog signal $X_{3}$.

maximum SNR values corresponding to the most dominant well parameters of the three analog signals are all larger than the SNR values of the fixed potential well parameters. Therefore, for different input signals, it is crucial to determine the most dominant wells to obtain the best output of the system.

After determining the most dominant well parameters of the three analog signals, the SNR changes with the change of step size and underdamping factor are shown in Figure 6.

Figure 6 shows that, with the change of step size and underdamping factor, the corresponding SNR also changes significantly for different analog signals. The most optimal SNR and system output can be obtained by selecting the appropriate step size and underdamping factor.

\section{Case Studies with Different Fault Types}

A machinery fault simulator is used for case analysis in this paper, as shown in Figure 7.

The testing bearings are installed on the test bench, and the bearing information is shown in Table 1. The accelerometers are placed on the bearing seats of the bearings under test, and the vibration signals are collected by the vibration 


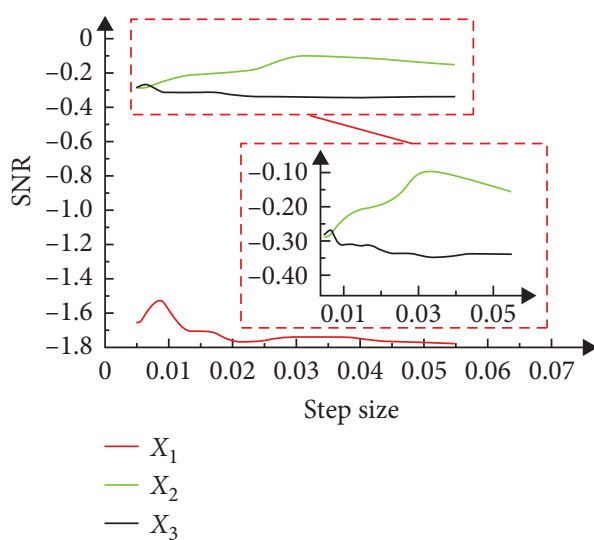

(a)

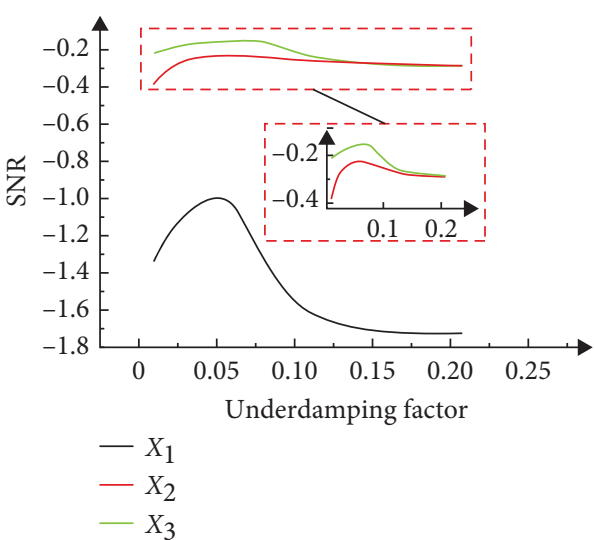

(b)

Figure 6: The trend of SNR with (a) step size and (b) underdamping factor.

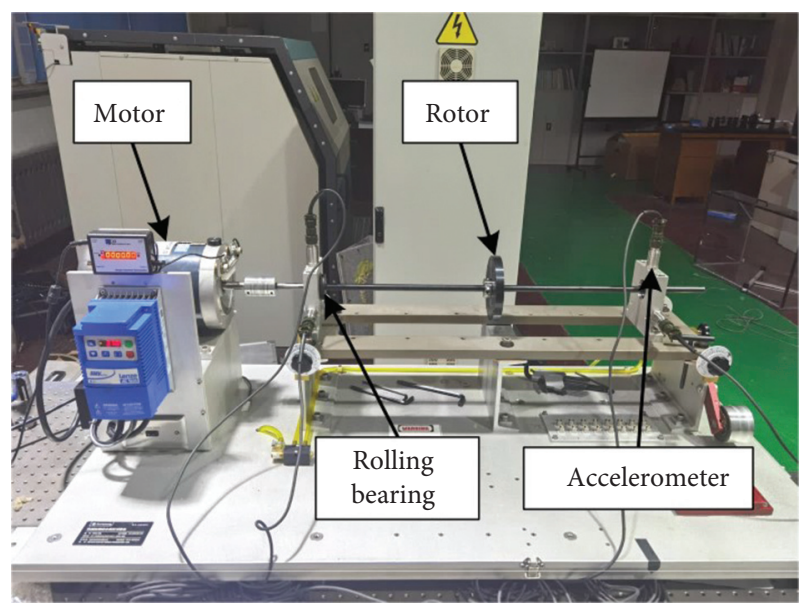

Figure 7: The machinery fault simulator.

data collector. The sampling frequency is $16,384 \mathrm{~Hz}$, and data points are 81,920. The formulas for calculating fault characteristic frequency $\left(f_{c f}\right)$ of rolling bearings are as follows:

$$
\begin{aligned}
B P F O & =\left(\frac{N_{1}}{2}\right) n_{1}\left[1-\left(\frac{d}{D_{1}}\right) \cos \varphi\right], \\
B P F I & =\left(\frac{N_{1}}{2}\right) n_{1}\left[1+\left(\frac{d}{D_{1}}\right) \cos \varphi\right], \\
B S F & =\left(\frac{N_{1}}{2}\right)\left(\frac{D_{1}}{d}\right)\left\{1-\left[\frac{d}{D_{1}} \cos \varphi\right]^{2}\right\},
\end{aligned}
$$

where $B P F O, B P F I$, and $B S F$ represent $f_{c f}$ of the outer ring, inner ring, and rolling element, respectively, $d$ and $n_{1}$ represent the diameter and number of rolling elements, respectively, $D_{1}$ is the pitch diameter, $\varphi$ is the contact angle, and $N_{1}$ is the rotational speed of the shaft.

According to the relevant parameters of bearings, Table 2 shows the theoretical fault characteristic frequencies of rolling bearings.
TABLE 1: Information of the tested bearings.

\begin{tabular}{lccc}
\hline $\begin{array}{l}\text { Number of } \\
\text { rolling elements }\end{array}$ & $\begin{array}{c}\text { Rolling element } \\
\text { diameter (inch) }\end{array}$ & $\begin{array}{c}\text { Pitch } \\
\text { diameter } \\
\text { (inch) }\end{array}$ & $\begin{array}{c}\text { Contact } \\
\text { angle }\end{array}$ \\
\hline 8 & 0.3125 & 1.319 & 0 \\
\hline
\end{tabular}

TABLE 2: Fault characteristic frequencies of rolling bearings.

\begin{tabular}{lccc}
\hline Speed of the motor $(\mathrm{r} / \mathrm{min})$ & $B P F O(\mathrm{~Hz})$ & $B P F I(\mathrm{~Hz})$ & $B S F(\mathrm{~Hz})$ \\
\hline 1200 & 61.05 & 98.95 & 39.84 \\
2400 & 122.09 & 197.91 & 79.68 \\
4800 & 244.19 & 395.82 & 159.36 \\
\hline
\end{tabular}

4.1. Outer Ring Fault Detection. The outer ring fault is used as a case, which is set in the outer raceway of the bearing. The speed of the motor is $2400 \mathrm{r} / \mathrm{min}$. Figure 8 shows the time and frequency domain graphs of the original vibration signal.

As can be seen from Figure 8, the waveform is not periodic, the fault features of rolling bearings are not obvious, the peaks corresponding to the rotational frequency $\left(f_{c}\right)$ and double rotational frequency $\left(2 f_{c}\right)$ are more obvious, there are a large number of mixed peaks, and the weak fault features of rolling bearings are not easy to extract, and they can easily lead to misdiagnosis. The proposed USAGVSR method is used to process the vibration signal and compared with the stochastic resonance, time-delayed feedback stochastic resonance (TFSR), and underdamped second-order stochastic resonance methods as follows.

4.1.1. Stochastic Resonance. At present, most of the existing SR methods fix the potential well parameters as specific values to simplify the complexity of the system and improve the efficiency of calculation. The potential well parameters are fixed as $a_{2}=1$ and $b_{2}=0.2$, the collected vibration signal is preprocessed, and the carrier frequency $f_{c 1}=100 \mathrm{~Hz}$ is set for modulation processing. Then, the SR system deals with the processed vibration signal, and the step size is optimized by ant colony algorithm. When $\mathrm{SNR}=-0.5710 \mathrm{~dB}$, the best 


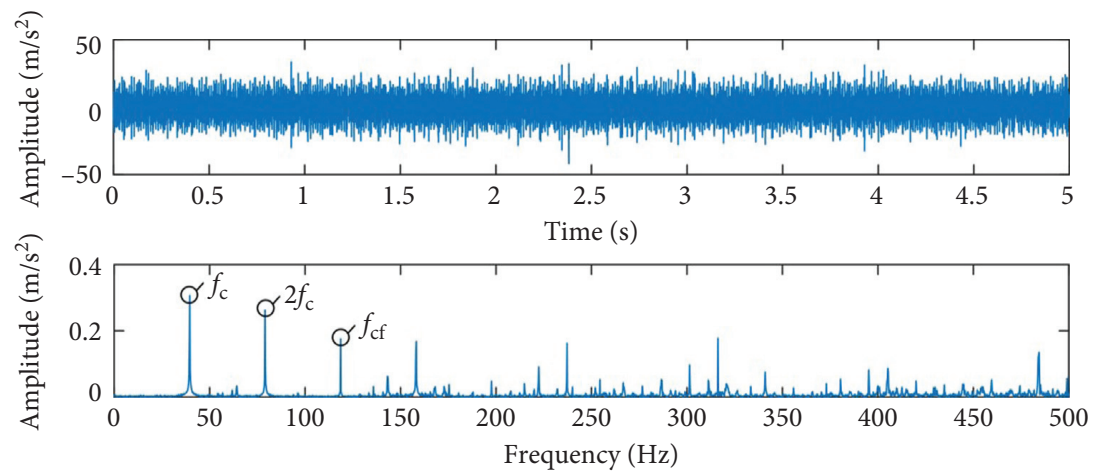

FIgURE 8: Original vibration signal.

result is obtained, $h=0.035$, and then the optimal output signal is demodulated. Figure 9 shows the time and frequency domain graphs processed by the SR system.

As can be seen from Figure 9, the hybrid peak value of the vibration signal processed by the SR system is weakened, but the peaks corresponding to $f_{c}$ and $2 f_{c}$ are more obvious. Moreover, there is an obvious peak value at the inner ring fault characteristic frequency, which easily leads to the misidentification of bearing fault types.

4.1.2. Time-Delayed Feedback Stochastic Resonance. The potential well parameters are fixed as $a_{2}=1$ and $b_{2}=0.2$. The TFSR system is used to process the vibration signal, and ant colony algorithm is used to optimize the step size $h$ and feedback strength $\beta$. When SNR $=-0.5821 \mathrm{~dB}, h=0.027$, and $\beta=0.035$, the best output signal is obtained. Figure 10 shows the time and frequency domain graphs processed by the TFSR system.

As can be seen from Figure 10, the vibration signal processed by the TFSR system still has a large number of hybrid peaks, and the corresponding peaks of $f_{c}$ and $2 f_{c}$ are obvious, which is not conducive to the extraction of rolling bearing weak fault features.

4.1.3. Underdamped Second-Order Stochastic Resonance. In the same way, the potential well parameters are fixed as $a_{2}=1$ and $b_{2}=0.2$. The USSR system is used to process the vibration signal, and the underdamped factor and step size are optimized by ant colony algorithm. When $\mathrm{SNR}=-0.533 \mathrm{~dB}, h=0.004$, and $k=0.14$, the best output signal is obtained. Figure 11 shows the time and frequency domain graphs processed by the USSR system.

Figure 11 indicates that the signal waveform processed by the USSR system is periodic, the corresponding peak of $2 f_{c}$ is obvious, and the corresponding peak value at $f_{c f}$ is also evident.

4.1.4. Underdamped Second-Order Adaptive General Variable-Scale Stochastic Resonance. On the basis of the USSR, the adaptive general variable-scale stochastic resonance is introduced, and the optimal barrier parameters are first made sure according to input signals. The collected vibration signal is preprocessed and then input into the adaptive general variable-scale stochastic resonance system. Set the scale coefficient $m=2000$ and $D=0.05$, and the dominant well parameters $a_{1}$ and $b_{1}$ are found by ant colony algorithm. When SNR is the largest, the optimal barrier and well parameters $a_{1}$ and $b_{1}$ are obtained, which are $\Delta U=0.004073$, $a_{1}=0.0315$, and $b_{1}=0.0609$, respectively. After the optimal barrier is determined, the USSR system processes the vibration signal, and the underdamped factor and step size are optimized by ant colony algorithm. The optimal system result is obtained when $\mathrm{SNR}=-0.0328 \mathrm{~dB}$, at which $h=0.0182$ and $k=0.86$. Figure 12 shows the time and frequency domain graphs processed by the USAGVSR system.

Figure 12 indicates that the signal waveform processed by the USAGVSR system has an obvious periodicity. In the frequency domain graph, there is a peak at $f_{c}$, and compared with the above systems, the peak value at $f_{c f}$ is more obvious, and the SNR is also significantly improved. Therefore, the USAGVSR system has better effects, more weak fault features are extracted, and the fault types are accurately recognized.

4.2. Inner Ring Fault Detection. The fault is set in the inner raceway of the bearing. The speed of the motor is $2400 \mathrm{r} / \mathrm{min}$. The original vibration signal time and frequency domain graphs are shown in Figure 13. There is no periodicity in the waveform, the peak value corresponding to $f_{c}$ is obvious, the fault features are weak, and they are not easy to extract.

4.2.1. Stochastic Resonance. The potential well parameters are fixed as $a_{2}=1$ and $b_{2}=0.2$. The SR system is used to process the vibration signal, and ant colony algorithm is used to optimize the step size. When SNR $=-0.7629 \mathrm{~dB}$ and $h=0.043$, the best output signal is obtained. Figure 14 shows the time and frequency domain graphs processed by the SR system.

Figure 14 indicates that the peak value at $f_{\mathrm{c}}$ is obvious, the fault features are weak, and there are some chaotic peaks, which are not conducive to the extraction of fault information.

4.2.2. Time-Delayed Feedback Stochastic Resonance. The potential well parameters are fixed as $a_{2}=1$ and $b_{2}=0.2$. The TFSR system is used to process the vibration signal, and ant 


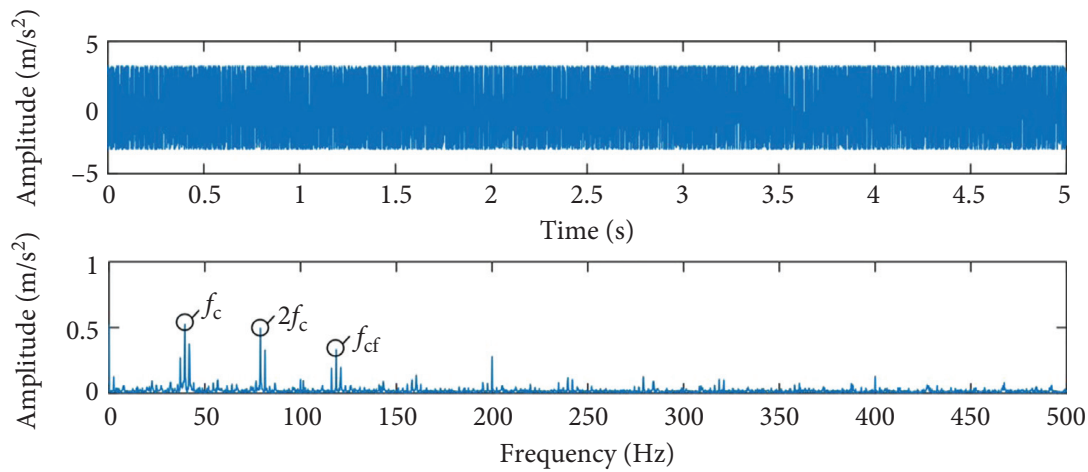

FIgURE 9: Signal processed by SR.

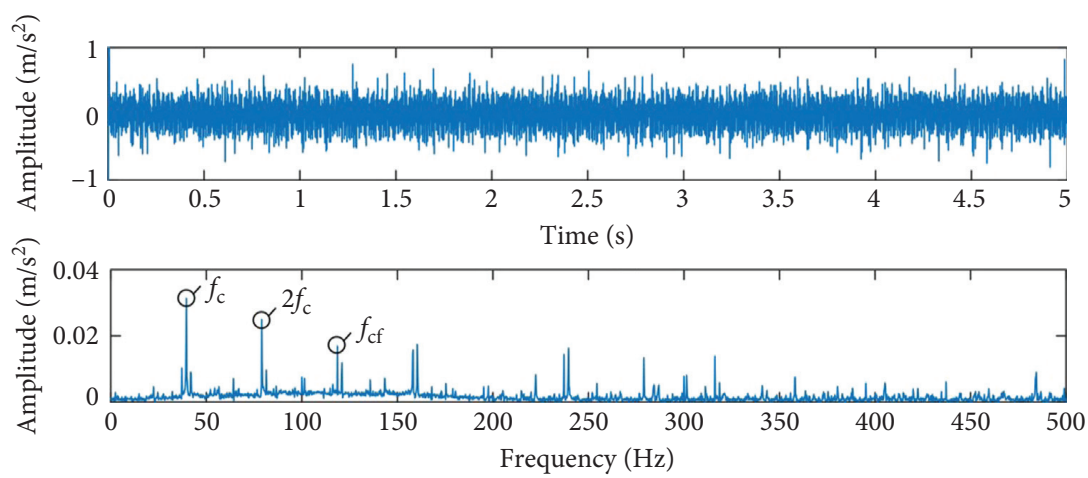

FIgURE 10: Signal processed by TFSR.

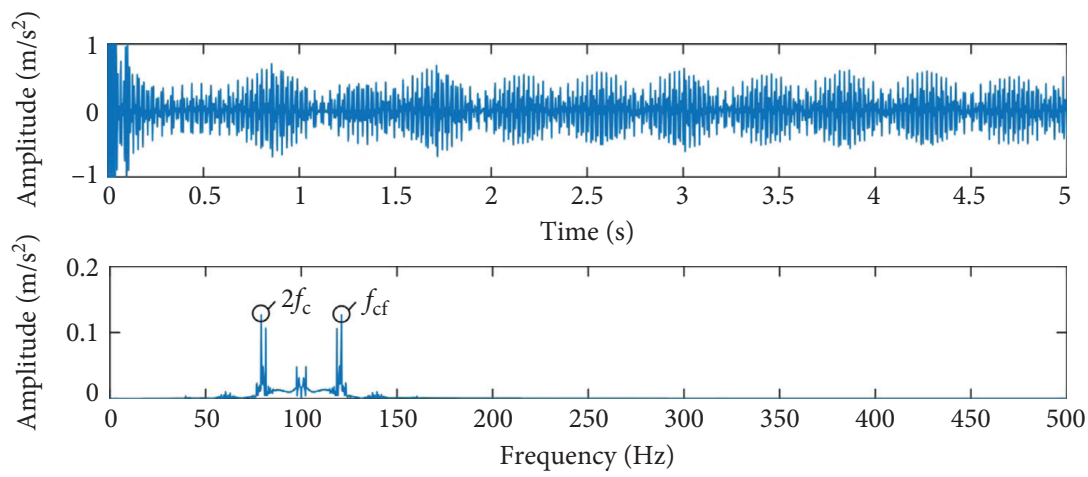

FIgURE 11: Signal processed by USSR.

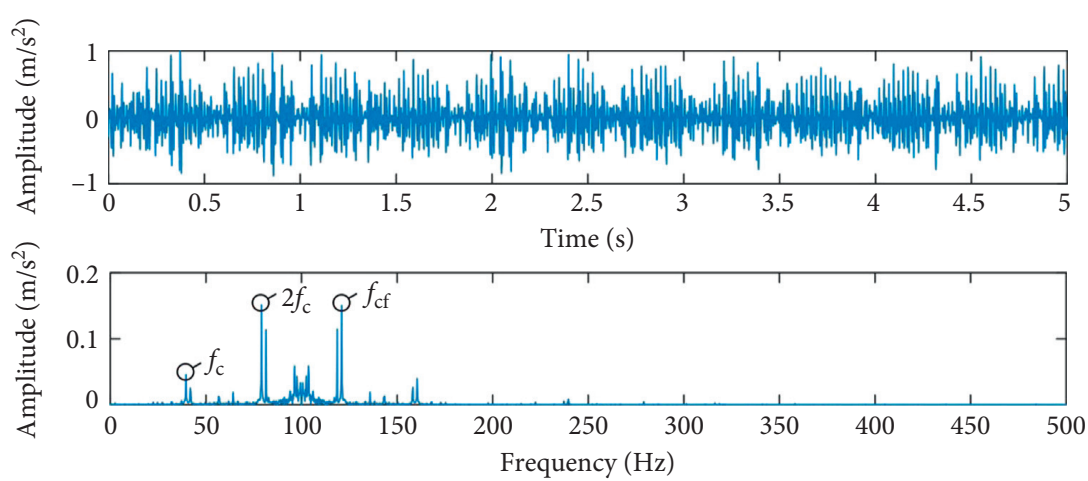

FIGURE 12: Signals processed by USAGVSR. 


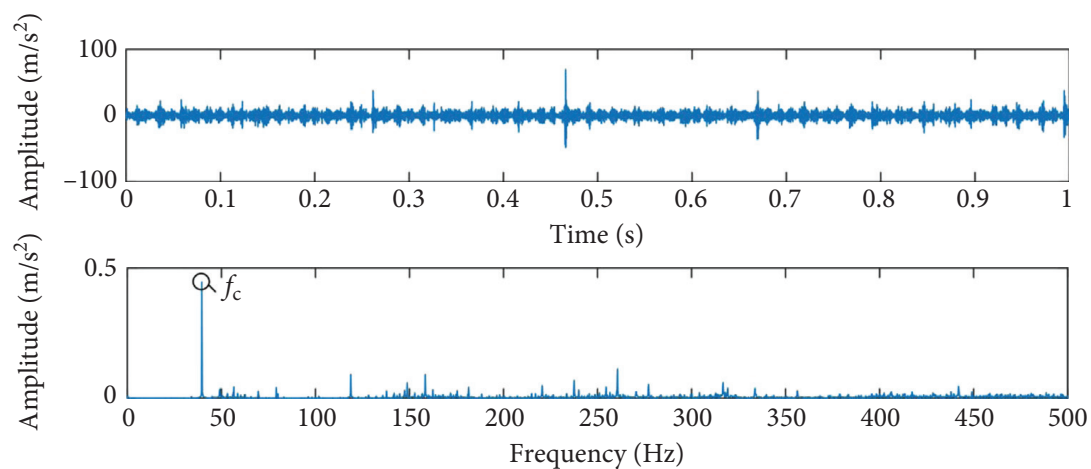

FIGURE 13: Original vibration signal.

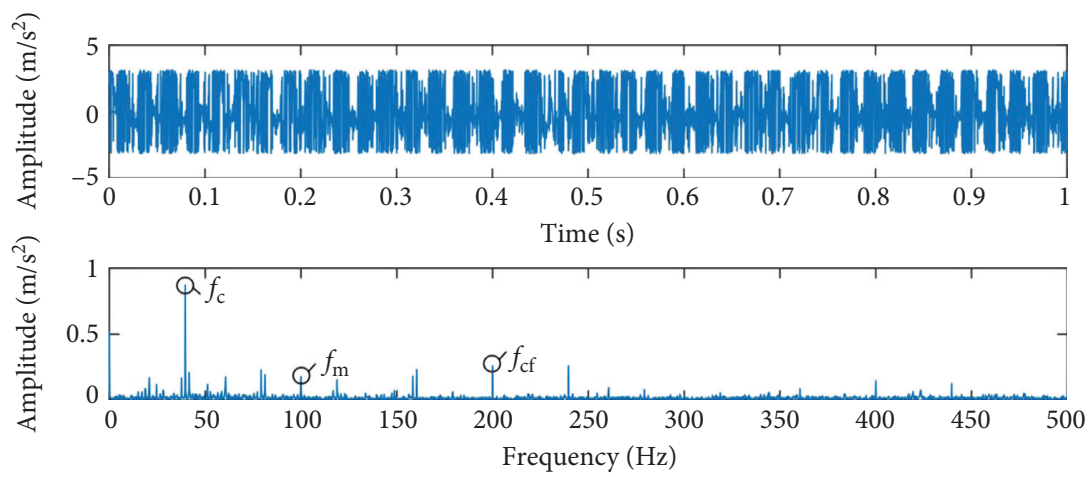

FIgURE 14: Signal processed by SR.

colony algorithm is used to optimize the step size and feedback strength. When $\mathrm{SNR}=-0.7751 \mathrm{~dB}, h=0.018$, and $\beta=0.024$, the best output signal is obtained. Figure 15 shows the time and frequency domain graphs processed by the TFSR system.

Figure 15 indicates that the vibration signal processed by the TFSR system has weak periodicity, the fault features are not obvious, and SNR is low, which is not conducive to the fault identification of rolling bearings.

\subsubsection{Underdamped Second-Order Stochastic Resonance.} Similarly, the parameters $a_{2}=1$ and $b_{2}=0.2$ are set, and the vibration signal is processed by the USSR system. When $\mathrm{SNR}=-0.765 \mathrm{~dB}$, the optimal system output is obtained, at which $h=0.0062$ and $k=0.75$. Figure 16 shows the time and frequency domain graphs processed by the USSR system.

Figure 16 indicates that the signal processed by the USSR system has a clear waveform graph, and the peak value at the modulation frequency is relatively obvious, so is the peak value at $f_{c f}$

4.2.4. Underdamped Second-Order Adaptive General Variable-Scale Stochastic Resonance. Similarly, the collected vibration signal is input into the adaptive general variablescale stochastic resonance system, and the corresponding most dominant barrier parameters are found by ant colony algorithm, which are $a_{1}=0.175, b_{1}=0.0625$, and $\Delta U=0.1225$, respectively. After the optimal potential barrier is determined, the vibration signal is input into the USSR system. When $\mathrm{SNR}=-0.4319 \mathrm{~dB}$, the optimal system output is obtained, at which $h=0.0058$ and $k=0.93$. Figure 17 shows the time and frequency domain graphs processed by the USAGVSR system.

Figure 17 indicates that the signal waveform graph processed by the USAGVSR system is clearer and has a more obvious periodicity. In the frequency domain graph, the amplitude at $f_{c f}$ is relatively evident, and compared with the processing results of the above systems, SNR is also significantly improved.

4.3. Rolling Element Fault Detection. In addition, the fault is set in the rolling element of the bearing. The speed of the motor is $2400 \mathrm{r} / \mathrm{min}$. Figure 18 shows the time and frequency domain graphs of the original vibration signal. It can be seen that there are many irregular pulse shocks on the waveform graph, and there are some high-energy spikes in the frequency domain graph, which may cause misdiagnosis of fault types.

4.3.1. Stochastic Resonance. The potential well parameters are fixed as $a_{2}=1$ and $b_{2}=0.2$. The SR system is used to process the vibration signal, and ant colony algorithm is used to optimize the step size. When $\mathrm{SNR}=-0.6003 \mathrm{~dB}$ and $h=0.0027$, the best output signal is obtained. Figure 19 shows the time and frequency domain graphs processed by the SR system. 


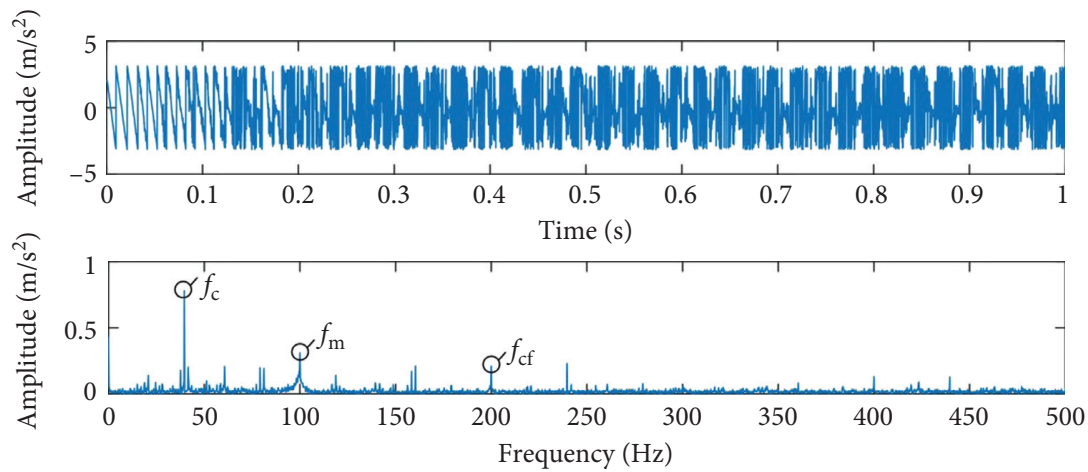

FIgURE 15: Signal processed by TFSR.

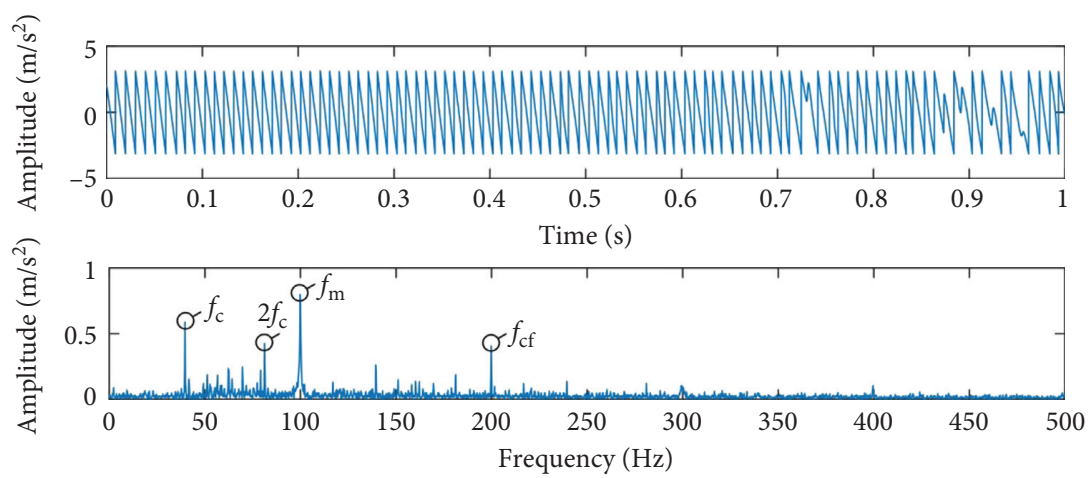

FIGURE 16: Signal processed by USSR.

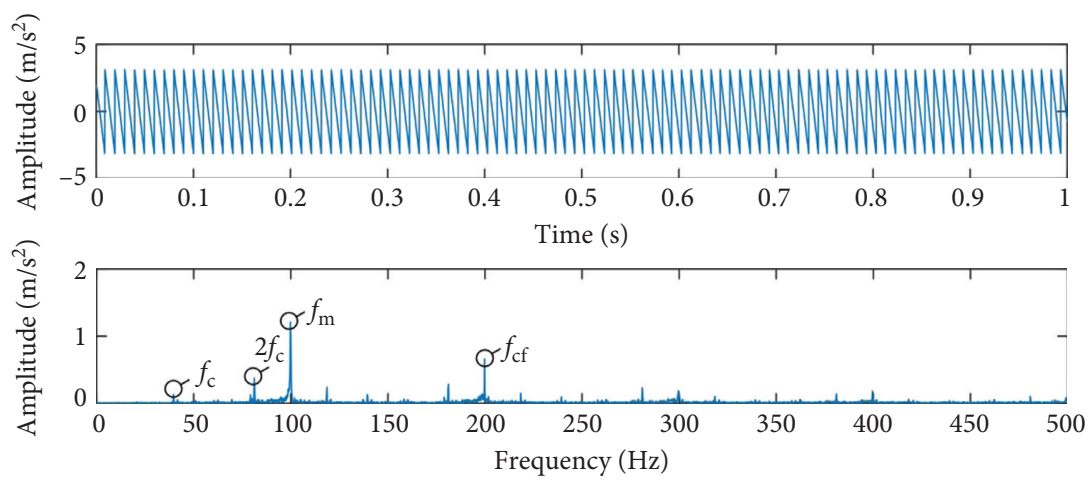

FIGURE 17: Signals processed by USAGVSR.

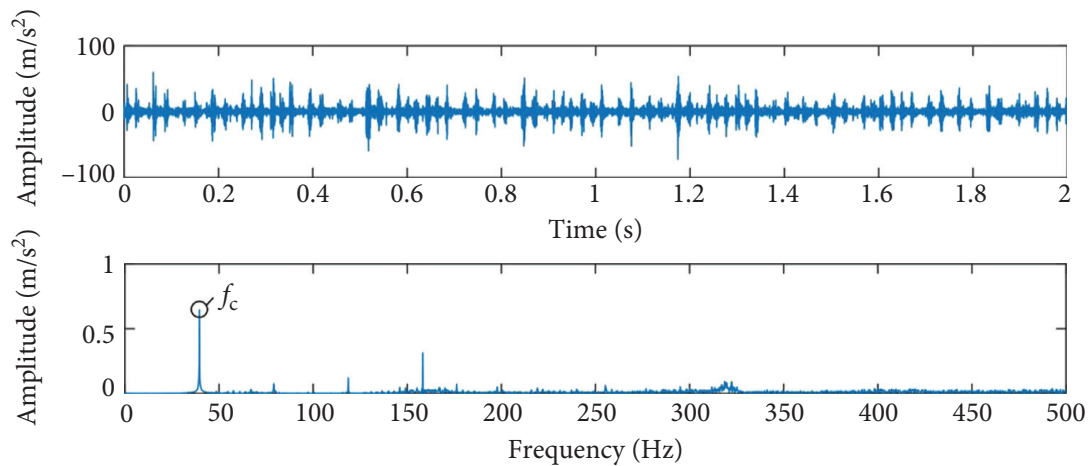

FIGURE 18: Original vibration signal. 


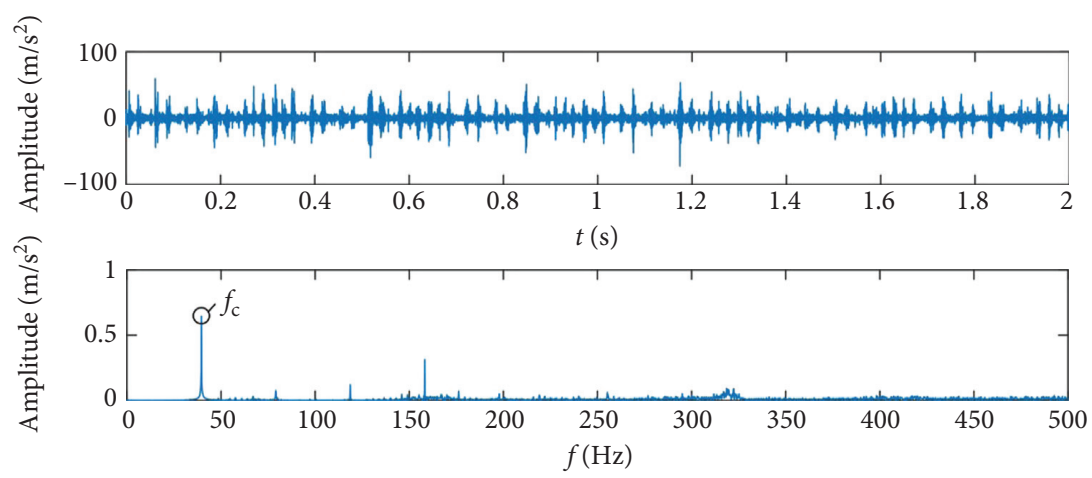

FIgURE 19: Signal processed by SR.

Figure 19 indicates that the peak value at $f_{c}$ is relatively obvious, the fault feature is weak, and the fault information of the rolling bearing cannot be extracted.

4.3.2. Time-Delayed Feedback Stochastic Resonance. The potential well parameters are fixed as $a_{2}=1$ and $b_{2}=0.2$. The TFSR system is used to process the vibration signal, and ant colony algorithm is used to optimize the step size and feedback strength. When $\mathrm{SNR}=-0.5969 \mathrm{~dB}, h=0.031$, and $\beta=0.079$, the best output signal is obtained. Figure 20 shows the time and frequency domain graphs processed by the TFSR system.

Figure 20 indicates that there is a high peak value at $f_{c}$, and the fault feature is not obvious. In addition, there are a large number of hybrid peaks at $f_{c f}$, and the fault identification of the rolling bearing cannot be carried out.

\subsubsection{Underdamped Second-Order Stochastic Resonance.} The parameters $a_{2}=1$ and $b_{2}=0.2$ are set, and the vibration signal is processed by the USSR system. When $\mathrm{SNR}=-0.5924 \mathrm{~dB}$, the optimal system output is obtained, at which $h=0.0079$ and $k=0.67$. Figure 21 shows the time and frequency domain graphs processed by the USSR system.

Figure 21 indicates that the signal processed by the USSR system has a peak at $f_{c f}$, which can diagnose the existence of the rolling element fault, but the corresponding amplitude at $f_{c}$ is relatively high.

4.3.4. Underdamped Second-Order Adaptive General Variable-Scale Stochastic Resonance. Similarly, the collected vibration signal is input into the adaptive general variablescale stochastic resonance system, and the corresponding most dominant barrier parameters are found by ant colony algorithm, which are $a_{1}=0.0875, b_{1}=0.0625$, and $\Delta U=0.030625$, respectively. After the optimal potential barrier is determined, the vibration signal is input into the USSR system. When $\mathrm{SNR}=-0.1355 \mathrm{~dB}$, the optimal system output is obtained, where $h=0.014$ and $k=0.40$. Figure 22 shows the time and frequency domain graphs processed by the USAGVSR system.

Figure 22 indicates that the signal waveform graph processed by the USAGVSR system is clearer and has a more obvious periodicity. Compared with the processing results of the above systems, the amplitude at $f_{c}$ is significantly decreased, the amplitude at $f_{c f}$ is obviously increased, and SNR is also significantly improved. Therefore, the USAGVSR method has a better effect and can precisely identify the fault types of bearings.

\section{Case Studies with Different Speeds}

The outer ring fault is used as a case, the rotational speed of the shaft is set to $1200 \mathrm{r} / \mathrm{min}, 2400 \mathrm{r} / \mathrm{min}$, and $4800 \mathrm{r} / \mathrm{min}$, respectively, and the rotation speed of $2400 \mathrm{r} / \mathrm{min}$ is analyzed as above. The following is an analysis of the rotational speeds of $1200 \mathrm{r} / \mathrm{min}$ and $4800 \mathrm{r} / \mathrm{min}$.

5.1. Outer Ring Fault at $1200 \mathrm{r} / \mathrm{min}$. The original vibration signal time and frequency domain graphs are shown in Figure 23.

As can be seen from Figure 23, the amplitude of $f_{c f}$ of the original vibration signal is obvious, but there are some hybrid peaks, which are not conducive to the weak fault feature extraction of rolling bearings. The original vibration signal is processed by USSR and the proposed USAGVSR method, and the processing results are as follows.

5.1.1. Underdamped Second-Order Stochastic Resonance. The parameters $a_{2}=1$ and $b_{2}=0.2$ are set, and the vibration signal is processed by the USSR system. When SNR $=-4.0449 \mathrm{~dB}$, the optimal system output is obtained, at which $h=0.0162$ and $k=0.126$. Figure 24 shows the time and frequency domain graphs processed by the USSR system.

Figure 24 indicates that the signal processed by the USSR system has an obvious peak at $f_{c f}$, but there is still a high hybrid peak frequency, which is easy to cause misdiagnosis.

5.1.2. Underdamped Second-Order Adaptive General Variable-Scale Stochastic Resonance. Similarly, the collected vibration signal is input into the adaptive general variablescale stochastic resonance system, and the corresponding most dominant barrier parameters are found by ant colony algorithm, which are $a_{1}=0.42, b_{1}=0.094$, and $\Delta U=0.4691$, respectively. After the optimal potential barrier is determined, the vibration signal is input into the USSR system. When $\mathrm{SNR}=-3.2370 \mathrm{~dB}$, the optimal system output is 


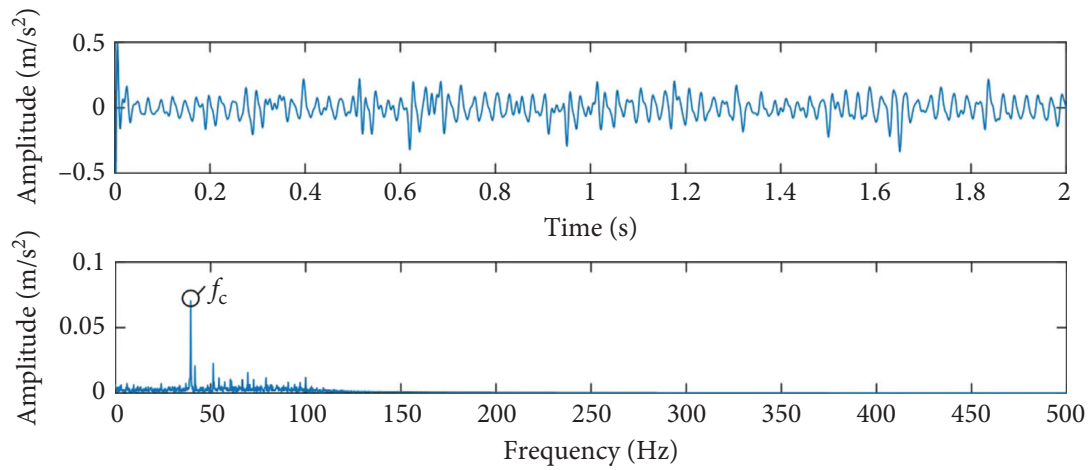

FIGURE 20: Signal processed by TFSR.

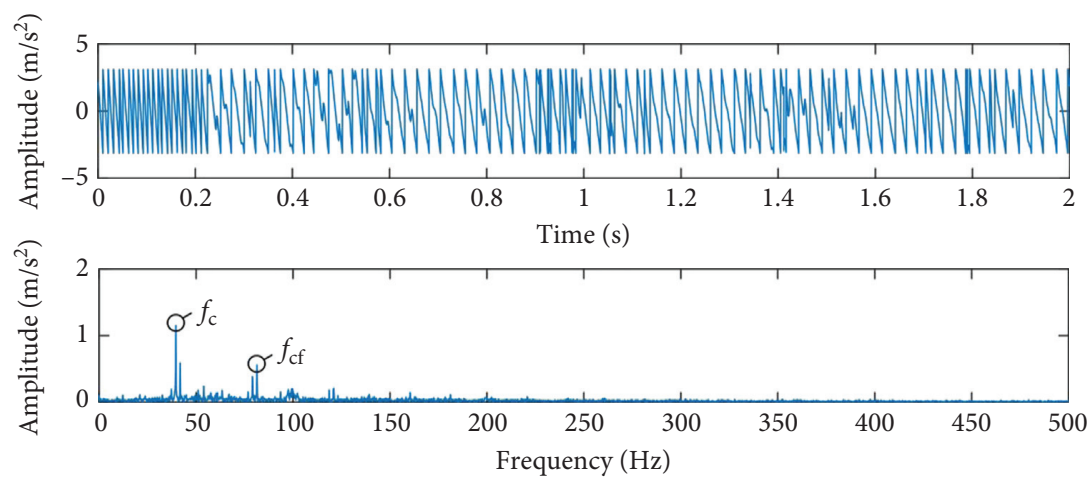

FIgURe 21: Signal processed by USSR.
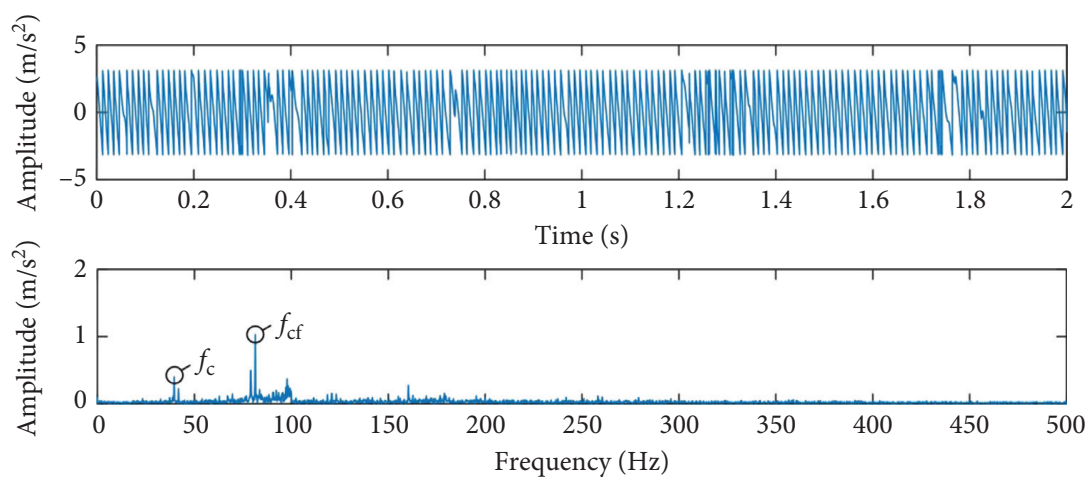

FIGURE 22: Signals processed by USAGVSR.

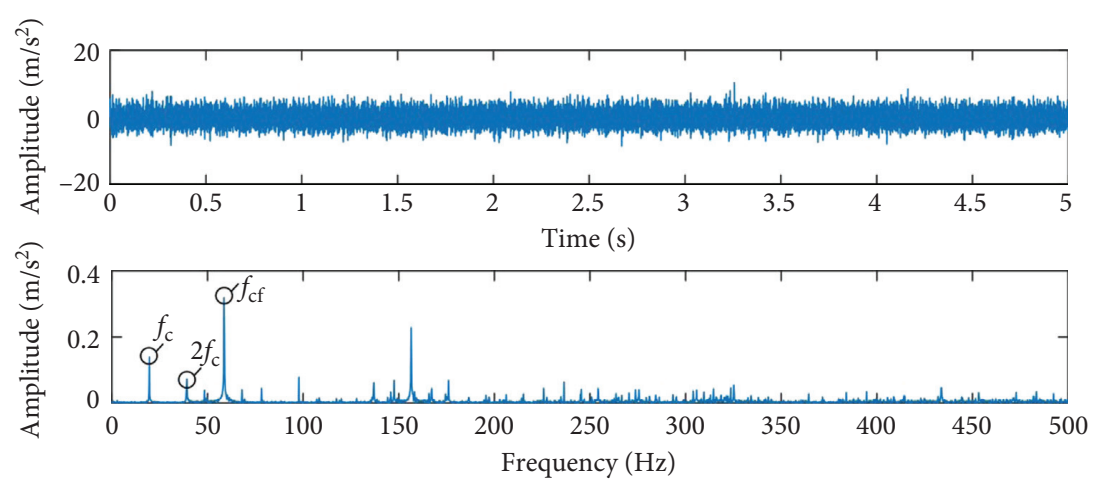

FIGURE 23: Original vibration signal. 


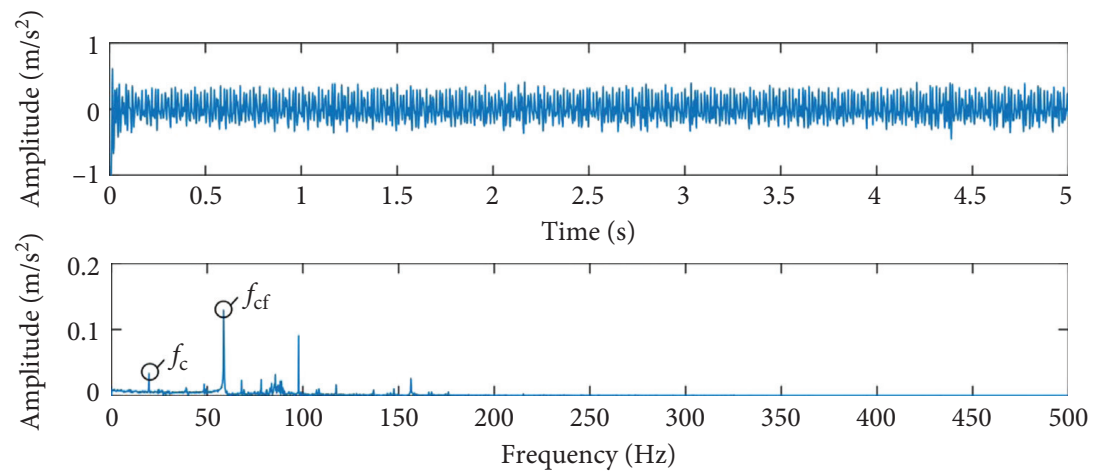

FIGURE 24: Signal processed by USSR.

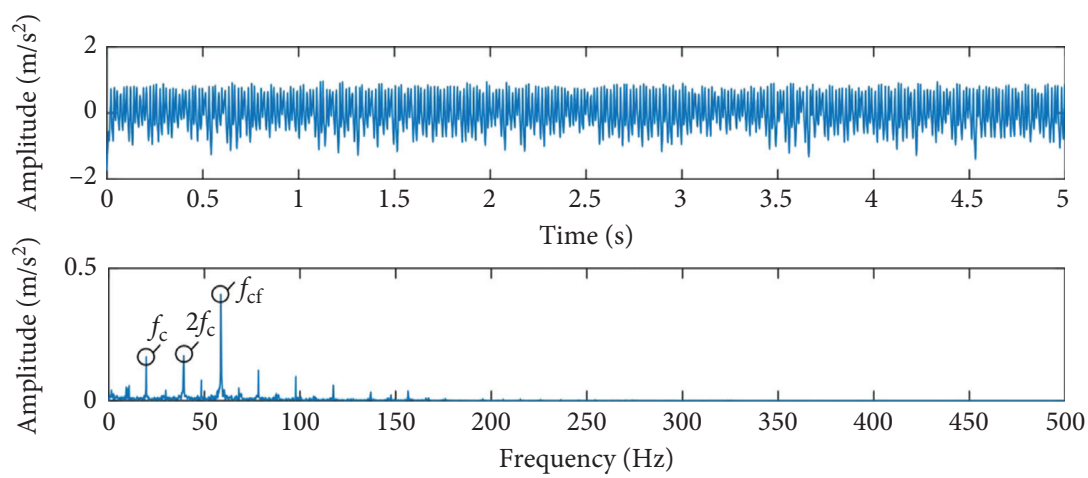

FIGURE 25: Signals processed by USAGVSR.

obtained, where $h=0.025$ and $k=0.643$. Figure 25 shows the time and frequency domain graphs processed by the USAGVSR system.

Figure 25 indicates that the amplitude at $f_{c f}$ of the signal processed by the USAGVSR system is obvious, the hybrid peak value is weakened, and the SNR is improved obviously.

5.2. Outer Ring Fault at $4800 \mathrm{r} / \mathrm{min}$. The original vibration signal time and frequency domain graphs are shown in Figure 26.

As can be seen from Figure 26, the $2 f_{c}$ amplitude of the original vibration signal is obvious, while the $f_{\mathrm{cf}}$ amplitude is the lower, which is not conducive to the weak fault feature extraction of rolling bearings. The original vibration signal is processed by USSR and the proposed USAGVSR method, and the processing results are as follows.

5.2.1. Underdamped Second-Order Stochastic Resonance. The parameters $a_{2}=1$ and $b_{2}=0.2$ are set, and the vibration signal is processed by the USSR system. When $\mathrm{SNR}=-1.226 \mathrm{~dB}$, the optimal system output is obtained, at which $h=0.01$ and $k=0.39$. Figure 27 shows the time and frequency domain graphs processed by the USSR system.

Figure 27 indicates that the signal processed by the USSR system has obvious peaks at $f_{\mathrm{c}}$ and $2 f_{\mathrm{c}}$, but $f_{\mathrm{cf}}$ cannot be extracted, which is not conducive to the fault diagnosis of rolling bearings.
5.2.2. Underdamped Second-Order Adaptive General Variable-Scale Stochastic Resonance. Similarly, the collected vibration signal is input into the adaptive general variablescale stochastic resonance system, and the corresponding most dominant barrier parameters are found by ant colony algorithm, which are $a_{1}=0.467, b_{1}=0.018$, and $\Delta U=3.029$, respectively. After the optimal potential barrier is determined, the vibration signal is input into the USSR system. When $\mathrm{SNR}=-0.4633 \mathrm{~dB}$, the optimal system output is obtained, where $h=0.008$ and $k=0.289$. Figure 28 shows the time and frequency domain graphs processed by the USAGVSR system.

Figure 28 indicates that the amplitudes at $2 f_{c}$ and $f_{c f}$ of the signal processed by the USAGVSR system are obvious, and the SNR is improved obviously, which is beneficial to extracting the fault characteristic information of rolling bearings. Therefore, the USAGVSR method has a better effect and can precisely identify the fault types of bearings.

\section{Results and Discussion}

The USAGVSR method proposed in this paper can effectively extract the weak fault feature information of rolling bearings. For different input signals, the adaptive general variable-scale stochastic resonance system is used to match the optimal potential barriers, and the optimal potential well parameters $a_{1}$ and $b_{1}$ are determined. Then, the signal is processed by the USSR system, and the parameters are adjusted by ant colony algorithm to get the 


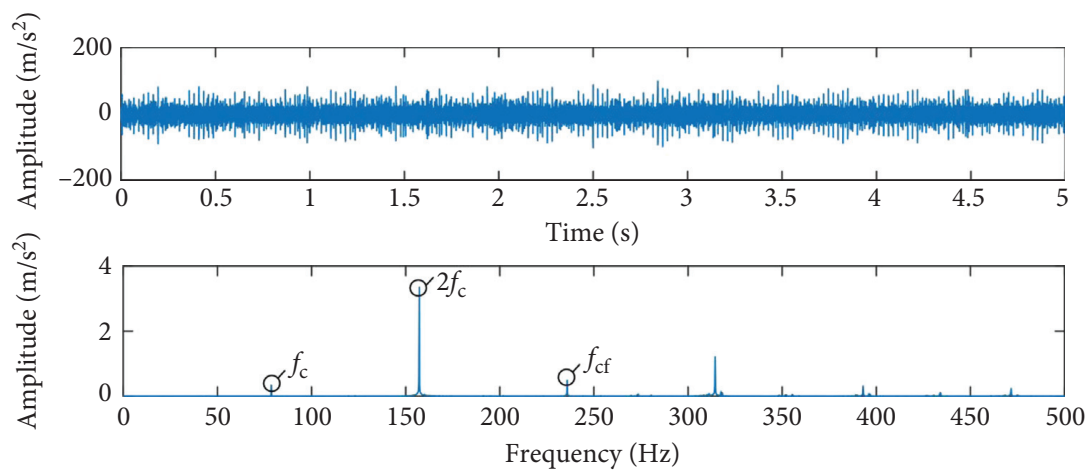

FIGURE 26: Original vibration signal.
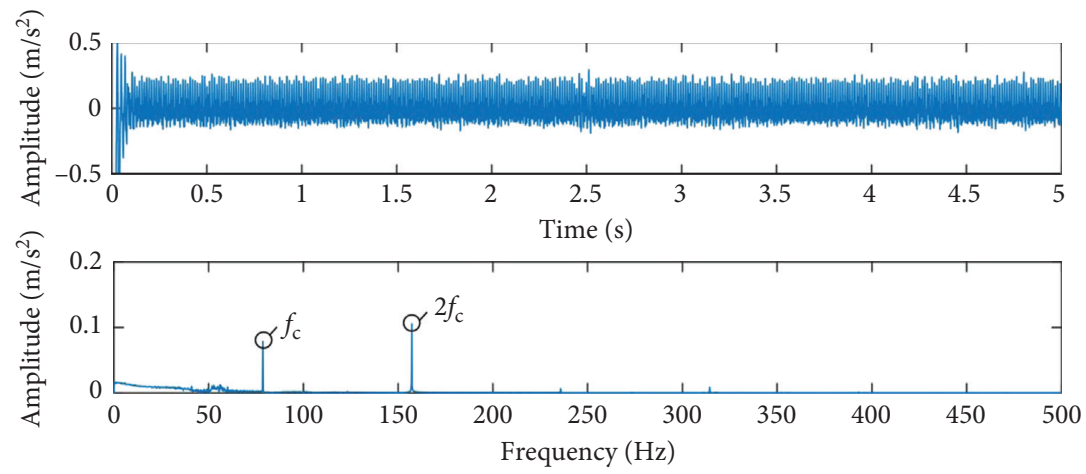

FIgURe 27: Signal processed by USSR.

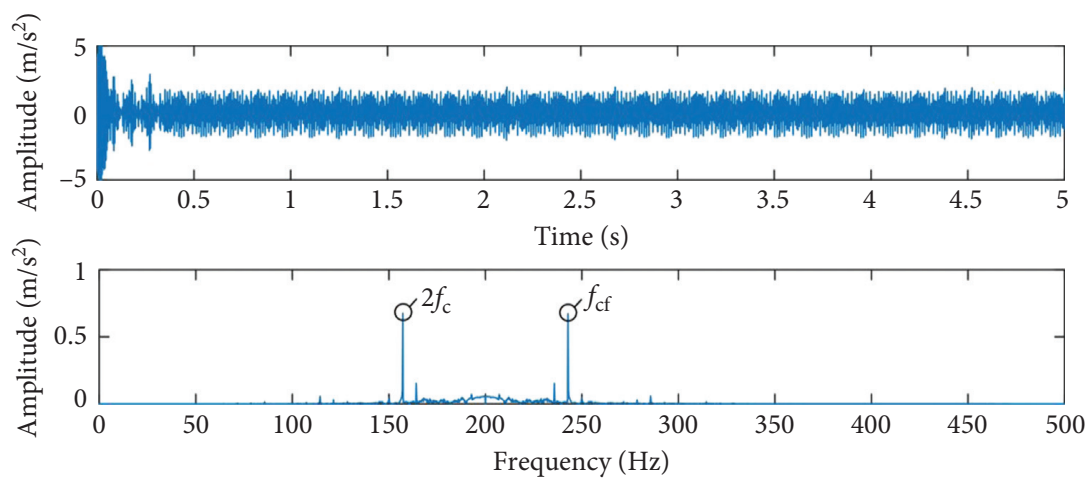

FIGURE 28: Signals processed by USAGVSR.

optimal system output. When the fault types are different, the comparison between the proposed method and the results of the above three systems is shown in Table 3. The comparison between the method proposed in this paper and the USSR method under different working conditions is shown in Table 4.

According to different fault types of rolling bearings and the faults under different working conditions, compared with the other methods, the SNR of the USAGVSR method is significantly improved. For different input signals, the USAGVSR method makes the transition frequency of the Brownian particle and the frequency of the input signal match best and determines the corresponding most advantageous barrier, which is the basis of subsequent SR processing. On the basis of the most dominant barrier,
TABle 3: Output SNR comparison of different fault types.

\begin{tabular}{lcccc}
\hline & $\begin{array}{c}\text { Output } \\
\text { SNR of SR } \\
(\mathrm{dB})\end{array}$ & $\begin{array}{c}\text { Output SNR } \\
\text { of TFSR } \\
(\mathrm{dB})\end{array}$ & $\begin{array}{c}\text { Output SNR } \\
\text { of USSR } \\
(\mathrm{dB})\end{array}$ & $\begin{array}{c}\text { Output SNR of } \\
\text { USAGVSR }(\mathrm{dB})\end{array}$ \\
\hline BPFO & -0.5710 & -0.5821 & -0.5330 & -0.0328 \\
$B P F I$ & -0.7629 & -0.7751 & -0.7650 & -0.4319 \\
$B S F$ & -0.6003 & -0.5969 & -0.5924 & -0.1355 \\
\hline
\end{tabular}

the optimal output of the SR is achieved. For different fault types and faults under different working conditions, the signals processed by this method have an obvious peak value at $f_{c f}$, which can better extract weak fault information, obtain the optimal output results, and accurately recognize the fault types. 
TABLE 4: Output SNR comparison of different rotation speeds.

\begin{tabular}{lccc}
\hline & Output SNR of USSR $(\mathrm{dB})$ & Output SNR of USAGVSR $(\mathrm{dB})$ & SNR increment $(\mathrm{dB})$ \\
\hline $1200 \mathrm{r} / \mathrm{min}$ & --4.0449 & -3.2370 & 0.8079 \\
$2400 \mathrm{r} / \mathrm{min}$ & -0.5330 & -0.032 & 0.5002 \\
$4800 \mathrm{r} / \mathrm{min}$ & -1.2260 & -0.4633 & 0.7627 \\
\hline
\end{tabular}

\section{Conclusion}

The potential well parameters' optimization on USAGVSR is conducted for weak fault detection of rolling bearings. The main findings are as follows:

(1) This method is compared with traditional methods with fixed potential well parameters, and the SNR is significantly improved, the fault characteristics of the output waveform are more obvious, and the bearing fault types are easier to identify

(2) According to different input signals, the corresponding barriers are adaptively matched, which lays a foundation for SR processing, and it is easier to obtain the best system output

(3) The method extracts the weak fault features of rolling bearings, which is beneficial to fault identification and accurate determination of fault types

The USAGVSR method has a good application prospect in rolling bearing fault diagnosis. In future research, we will combine the advantages of the proposed method with other fault diagnosis methods to carry out weak fault feature recognition of rolling bearings.

\section{Data Availability}

The data used to support the findings of this study are included within the article.

\section{Conflicts of Interest}

The authors declare that there are no conflicts of interest regarding the publication of this paper.

\section{Acknowledgments}

This work was supported by the National Science Foundation of China (nos. 52075348, 51905357, 52005352, and 51805337), Key Laboratory of Vibration and Control of Aero-Propulsion System, Ministry of Education, Northeastern University (VCAME202001), and Natural Science Foundation of Liaoning Province (no. 2019-ZD-0654).

\section{References}

[1] H. B. Lin, F. T Wu, and G. L He, "Rolling bearing fault diagnosis using impulse feature enhancement and nonconvex regularization," Mechanical Systems and Signal Processing, vol. 142, Article ID 106790, 2020.

[2] H. T. Shi, Y. Y. Li, X. T. Bai et al., "Investigation of the orbitspinning behaviors of the outer ring in a full ceramic ball bearing-steel pedestal system in wide temperature ranges,"
Mechanical Systems and Signal Processing, vol. 149, Article ID 107317, 2021.

[3] F. Jiang, K. Ding, G. L. He, and C. Y. Du, "Sparse dictionary design based on edited cepstrum and its application in rolling bearing fault diagnosis," Journal of Sound and Vibration, vol. 490, Article ID 115704, 2021.

[4] H. Li, H. Y. Lv, H. Sun et al., "Nonlinear vibrations of fiberreinforced composite cylindrical shells with bolt loosening boundary conditions," Journal of Sound and Vibration, vol. 496, Article ID 115935, 2021.

[5] M. S. P. Reddy, D. M. Reddy, S. Devendiran, and A. T. Mathew, "Bearing fault diagnosis using empirical mode decomposition, entropy based features and data mining techniques," Materials Today: Proceedings, vol. 5, no. 5, pp. 11460-11475, 2018.

[6] A. Glowacz, "Recognition of acoustic signals of induction motor using Fft, Smofs-10 and LSVM," Eksploatacja $i$ Niezawodność, vol. 17, no. 4, 2015.

[7] K. Yu, H. Ma, T. R. Lin, X. Li, and Q. Fu, "Simulation model guided weakly supervised adversarial domain adaptation approach for intelligent cross-machine fault diagnosis," Structural Health Monitoring, 2020.

[8] D. F. Zhao, S. L. Liu, D. Gu et al., "Improved multi-scale entropy and it's application in rolling bearing fault feature extraction," Measurement, vol. 152, Article ID 107361, 2020.

[9] S. Sun, K. Przystupa, M. Wei et al., "Fast bearing fault diagnosis of rolling element using Lévy Moth-Flame optimization algorithm and Naive Bayes," Eksploatacja I Niezawodność, vol. 22, no. 4, 2020.

[10] K. X. Shao, W. L. Fu, J. W. Tan, and K. Wang, "Coordinated approach fusing time-shift multiscale dispersion entropy and vibrational Harris hawks optimization-based SVM for fault diagnosis of rolling bearing," Measurement, vol. 173, Article ID 108580, 2021.

[11] A. Glowacz, W. Glowacz, J. Kozik et al., "Detection of deterioration of three-phase induction motor using vibration signals," Measurement Science Review, vol. 19, pp. 241-249, 2019.

[12] M. X. Hou and H. T. Shi, "Stator-winding incipient shortedturn fault detection for motor system in motorized spindle using modified interval observers," IEEE Transactions on Instrumentation and Measurement, vol. 70, Article ID 3505716, 2021.

[13] Y. Z. Yang, C. Liu, D. X. Jiang, and K. Behdinan, "Nonlinear vibration signatures for localized fault of rolling element bearing in rotor-bearing-casing system," International Journal of Mechanical Sciences, vol. 173, Article ID 105449, 2020.

[14] K. Kumar, S. Shukla, and S. K. Singh, "A combined approach for weak fault signature extraction of rolling element bearing using Hilbert envelop and zero frequency resonator," Journal of Sound and Vibration, vol. 419, pp. 436-451, 2018.

[15] H. X. Zhou, H. Li, T. Liu, and Q. Chen, "A weak fault feature extraction of rolling element bearing based on attenuated cosine dictionaries and sparse feature sign search," ISA Transactions, vol. 97, pp. 143-154, 2020. 
[16] J. Dybala and R. Zimroz, "Rolling bearing diagnosing method based on Empirical Mode Decomposition of machine vibration signal," Applied Acoustics, vol. 77, pp. 195-203, 2014.

[17] J. Singh, A. K. Darpe, and S. P. Singh, "Rolling element bearing fault diagnosis based on Over-Complete rational dilation wavelet transform and auto-correlation of analytic energy operator," Mechanical Systems and Signal Processing, vol. 100, pp. 662-693, 2018.

[18] G. M. Dong and J. Chen, "Noise resistant time frequency analysis and application in fault diagnosis of rolling element bearings," Mechanical Systems and Signal Processing, vol. 33, pp. 212-236, 2012.

[19] X. Y. Zhang and J. Z. Zhou, "Multi-fault diagnosis for rolling element bearings based on ensemble empirical mode decomposition and optimized support vector machines," $\mathrm{Me}$ chanical Systems and Signal Processing, vol. 41, pp. 127-140, 2013.

[20] W. Cheng, X. M. Xu, Y. P. Ding et al., "An adaptive smooth unsaturated bistable stochastic resonance system and its application in rolling bearing fault diagnosis," Chinese Journal of Physics, vol. 65, pp. 629-641, 2020.

[21] S. Wang, P. J. Niu, Y. F. Guo et al., "Early diagnosis of bearing faults using decomposition and reconstruction stochastic resonance system," Measurement, vol. 158, Article ID 107709, 2020.

[22] C. B. He, P. Niu, R. Yang et al., "Incipient rolling element bearing weak fault feature extraction based on adaptive second-order stochastic resonance incorporated by mode decomposition," Measurement, vol. 145, pp. 687-701, 2019.

[23] G. Zhang, Y. L. Yang, and T. Q. Zhang, "The characteristic analysis of stochastic resonance and bearing fault diagnosis based on NWSG model driven by trichotomous noise," Chinese Journal of Physics, vol. 60, pp. 107-121, 2019.

[24] Z. X. Li and B. Q. Shi, "A piecewise nonlinear stochastic resonance method and its application to incipient fault diagnosis of machinery," Chinese Journal of Physics, vol. 59, pp. 126-137, 2019.

[25] Q. B. He, E. H. Wu, and Y. Y. Pan, "Multi-Scale Stochastic Resonance Spectrogram for fault diagnosis of rolling element bearings," Journal of Sound and Vibration, vol. 420, pp. 174-184, 2018.

[26] J. M. Li, J. F. Zhang, M. Li, and Y. G. Zhang, "A novel adaptive stochastic resonance method based on coupled bistable systems and its application in rolling bearing fault diagnosis," Mechanical Systems and Signal Processing, vol. 114, pp. 128145, 2019.

[27] Y. G. Lei, Z. J. Qiao, X. F. Xu, J. Lin, and S. T. Niu, “An underdamped stochastic resonance method with stable-state matching for incipient fault diagnosis of rolling element bearings," Mechanical Systems and Signal Processing, vol. 94, pp. 148-164, 2017.

[28] J. M. Li, M. Li, and J. F. Zhang, "Rolling bearing fault diagnosis based on time-delayed feedback monostable stochastic resonance and adaptive minimum entropy deconvolution," Journal of Sound and Vibration, vol. 401, pp. 139-151, 2017.

[29] S. L. Lu, Q. B. He, and F. R. Kong, "Effects of underdamped step-varying second-order stochastic resonance for weak signal detection," Digital Signal Processing, vol. 36, pp. 93-103, 2015.

[30] Q. Ma, D. Huang, and J. Yang, "Adaptive stochastic resonance in second-order system with general scale transformation for weak feature extraction and its application in bearing fault diagnosis," Fluctuation and Noise Letters, vol. 17, Article ID 1850009, 2018.
[31] G. Zhang, T. Yi, T. Zhang et al., "A multiscale noise tuning stochastic resonance for fault diagnosis in rolling element bearings," Chinese Journal of Physics, vol. 56, pp. 145-157, 2018.

[32] X. L. Zhang, W. Chen, B. J. Wang et al., "Intelligent fault diagnosis of rotating machinery using support vector machine with ant colony algorithm for synchronous feature selection and parameter optimization," Neurocomputing, vol. 167, pp. 260-279, 2015. 